\title{
Early-Onset Podocyte Injury and Glomerular Sclerosis in Osborne-Mendel Rats
}

\author{
Kyohei YASUNO ${ }^{1)}$, Shoko ISHIHARA ${ }^{1)}$, Rio SAITO'), Makoto ISHIKAWA ${ }^{1)}$, Takashi KATO ${ }^{1)}$, \\ Ryosuke KOBAYASHI ${ }^{1)}$, Tomoshige BABA ${ }^{1)}$, Kazuya KAWANO ${ }^{4}$, Kikumi OGIHARA ${ }^{2)}$, Junichi $\mathrm{KAMIIE}^{3)}$ and \\ Kinji SHIROTA ${ }^{1,3) *}$ \\ ${ }^{1)}$ Research Institute of Biosciences, ${ }^{2)}$ Laboratory of Environmental Pathology, School of Environmental Health and ${ }^{3)}$ Laboratory of \\ Veterinary Pathology, School of Veterinary Medicine, Azabu University, Kanagawa 229-8501 and ${ }^{4)}$ Tokushima Research Institute, \\ Otsuka Pharmaceutical Co., Ltd., Tokushima 771-0192, Japan
}

(Received 5 March 2010/Accepted 7 May 2010/Published online in J-STAGE 21 May 2010)

\begin{abstract}
Progressive glomerular injury associated with early-onset proteinuria was investigated in male Osborne-Mendel (OM) rats aged 5 to 20 weeks. Age-matched male Fischer 344 (F344) rats were used for comparison. OM rats developed mild hypertension and selective proteinuria (albuminuria) from 5 weeks of age, and non-selective proteinuria from 7 weeks of age. Light microscopy of OM kidney revealed hyaline droplets in the podocyte at 5 weeks of age and vacuolation of podocytes and adhesion of the capillary loop to the Bowman's capsule at 7 weeks of age. Segmental glomerulosclerosis developed in OM rats from 15 weeks of age, and global sclerosis appeared at 20 weeks of age. Desmin, a marker of podocye injury, was expressed in podocytes from 10 weeks of age, and the intensity of expression increased with age. Ultrastructurally, damage to podocytes such as effacement of foot processes, decreasing number of filtration slits, and rearrangement of the actin cytoskeleton were observed from 5 weeks of age in OM rat. Glomerular volume in OM rats increased with age and was consistently higher than in age-matched F344 rats. The number of WT-1-positive podocytes and vimentin-positive podocyte area were lower in OM rats and decreased with age. These findings suggest that glomerulonephropathy in male $\mathrm{OM}$ rats is associated with glomerular hypertrophy, progressive podocytopathy, and a reduction in podocyte number and area. Renal injury in OM rats was associated with development of early-onset proteinuria and was more progressive than in age-matched F344 rats. KEY WORDS: glomerulonephropathy, kidney, podocyte, proteinuria, rat.
\end{abstract}

Osborne-Mendel (OM) rats have long been used as obesity-prone rats [22]. When fed a high fat diet, they are susceptible to the development of obesity and demonstrate several metabolic abnormalities, including cardiac and renal hypertrophy, hyperglycemia, and hyperinsulinemia [4, 14]. These clinical symptoms are also characteristic of human metabolic syndrome, suggesting that $\mathrm{OM}$ rats could provide an animal model for human metabolic syndrome. Metabolic syndrome is a reported risk factor for progression of chronic kidney disease and for end-stage renal disease (ESRD) [23]. This time, characteristic progressive glomerular injury associated with early onset proteinuria in OM rats was found in our laboratory. However, to our knowledge, there have been no detailed pathological studies on renal disease, especially on glomerular injury in OM rats.

Podocytes are highly differentiated and specialized epithelial cells that line the outer aspect of the glomerular basement membrane (GBM). In the normal glomerulus, they serve as the final barrier against urinary protein loss. Foot processes of neighboring podocytes interdigitate over the capillary walls and are bridged by the slit diaphragms, thus forming the final filtration barrier. In proteinuric nephropathy, injuries to podocytes may develop in the early stage of the disease, and the ensuing reduction in podocyte number occurs continuously [20]. Detachment of podocytes from

\footnotetext{
* Correspondence to: Shirota, K., Laboratory of Veterinary Pathology, School of Veterinary Medicine, Azabu University, 117-71, Fuchinobe, Sagamihara, Kanagawa 229-8501, Japan. e-mail: shirota@azabu-u.ac.jp
}

the GBM results in a decrease in the number of glomerular podocytes and plays an important role in the development of severe proteinuria and progression of glomerular injury $[8$, $13,25]$. Because podocytes have very low potency to regenerate, loss of podocytes in the glomerulus leads to denudation of the GBM. This results in massive leakage of plasma proteins, adhesion of the glomerular capillary tuft to Bowman's capsule, and progression to segmental and global glomerular sclerosis [9].

Proteinuric nephropathies associated with progressive glomerular injury progress toward ESRD regardless of the type of primary glomerular lesion. Recent studies have revealed that podocytes are a key determinant in the maintenance of the permselective function of the glomerular capillary, and that both primary and secondary podocyte dysfunction are closely associated with proteinuria, glomerular sclerosis, and ESRD [6, 12, 20, 26].

In the present study, glomerular injury and the relationship between podocyte injury and the development of proteinuria and glomerulosclerosis were examined in young male OM rats. Fischer 344 (F344) rats, which are used for long-term toxicological tests, were used for comparison as they commonly develop chronic nephropathy in old age [24].

\section{MATERIALS AND METHODS}

Animals: All procedures in this study were in accordance with the guidelines approved by the Animal Research Com- 
mittee of our institution. Male $\mathrm{OM}(\mathrm{Om} / \mathrm{NSlc})$ rats $(\mathrm{n}=43)$ and male F344/NSlc rats ( $n=12$ ) were used (Japan SLC, Shizuoka, Japan). All rats were housed in a barrier system animal facility with a 12-hr light/dark cycle, temperature of 21 $\pm 1^{\circ} \mathrm{C}$, and relative humidity of 50 to $60 \%$. Rats were fed CLEA Rodent Diet CE-2 (CLEA Japan, Inc., Tokyo, Japan) with a sodium content of $0.36 \%$ and crude protein content of $25.1 \%$, and had free access to tap water. Both F344 and OM rats were housed in metabolic cages and 24-hr urine collection for protein excretion determination was conducted before necropsy. Systolic blood pressure of OM rats was measured by the tail plethysmography in awake animals at 7,13 and 20 weeks of age before necropsy. F344 rats were sacrificed at $5(n=3), 7(n=3), 10(n=3), 15(n=3)$ and 20 $(n=3)$ weeks of age and OM rats were sacrificed at $5(n=6)$, $7(\mathrm{n}=8), 10(\mathrm{n}=5), 12(\mathrm{n}=3), 13(\mathrm{n}=5), 15(\mathrm{n}=7), 17(\mathrm{n}=5)$ and $20(n=9)$ weeks of age. Histological changes in the cortical renal tissues were examined by light and electron microscopy as well as by immunohistochemistry. SDSpolyacrylamide gel electrophoresis (SDS-PAGE) was performed on urine samples $(1 \mu l)$ using $10 \%$ PAGE Bis-Tris gel. Gels were run at $40 \mathrm{~mA}$ for $90 \mathrm{~min}$ in Tris/glycine/SDS buffer (Bio-Rad Laboratories, Hercules, CA, U.S.A.) and proteins were visualized using Coomassie Brilliant Blue R250 (CBB R-250). Precision Plus Protein ${ }^{\mathrm{TM}}$ Standards (BioRad Laboratories) were used as molecular markers.

Morphological analysis of glomerulosclerosis: Kidney tissues obtained from F344 and OM rats were immediately fixed in $10 \%$ neutral buffered formaldehyde (NBF), dehydrated by graded alcohol, and embedded in paraffin for light microscopy. A single section $(3 \mu \mathrm{m})$ was stained with periodic acid-Schiff (PAS) in each animal and the presence of sclerosis in the glomerular tuft was investigated. Morphometric analysis was performed using a computer-based image analysis system (ImageJ, National Institutes for Health, Bethesda, MD, U.S.A.). For each kidney section, glomeruli were systematically encountered by moving the microscope stage with an S-shape path, and 50 glomeruli were digitally saved as 1,248 $\times 1,000$-pixel images. Exact enlargement ( $\mu \mathrm{m}$ per pixel) of the digital images was calculated from a reference grid digitized at the same resolution. The outline of the glomerular tuft area of each glomerulus was manually traced, and its area $\left(\mathrm{A}_{\mathrm{G}}\right)$ was automatically measured by ImageJ. The sclerotic area of each glomerulus $\left(A_{S}\right)$ was measured using the same method. The sclerosis score was then calculated as $\left(\mathrm{A}_{\mathrm{S}} / \mathrm{A}_{\mathrm{G}}\right) \times 100$.

Glomerular volume and glomerular density: Mean glomerular volume $\left(\mathrm{V}_{\mathrm{G}}\right)$ was calculated using the formula described by Nagata et al. [18]; $\mathrm{V}_{\mathrm{G}}=(\beta / \kappa) \times\left(\mathrm{A}_{\mathrm{G}}\right)^{2 / 3}$, where $\kappa=1.1$ (size distribution coefficient) and $\beta=1.38$ (shape coefficient for spheres). A total of 50 glomeruli was evaluated for each kidney section. Glomerular density was examined on kidney sections from 5-week-old rats, and was defined as glomerular number per unit area $\left(\mathrm{N}_{\mathrm{G}} / \mathrm{A}_{\mathrm{C}}\right)$. To establish this value, the number of glomeruli in the whole sections $\left(\mathrm{N}_{\mathrm{G}}\right)$ was counted, and the area of the kidney cortex $\left(\mathrm{A}_{\mathrm{C}}\right)$ was measured using ImageJ.
Immunohistochemistry: For immunohistochemistry, staining for desmin and vimentin was performed on $10 \%$ NBF-fixed, paraffin-embedded tissues, and staining for Wilms' Tumor-1 (WT-1) was performed on 4\% paraformaldehyde-fixed, paraffin-embedded kidney tissues. Sections (3 $\mu \mathrm{m})$ were heated in citrate buffer $(\mathrm{pH} \mathrm{6.0)}$ for $10 \mathrm{~min}$ at $90^{\circ} \mathrm{C}$ by microwave or $8 \mathrm{~min}$ at $121^{\circ} \mathrm{C}$ by autoclave, and the primary antibodies, mouse anti-desmin monoclonal antibody (1:100, clone ZC18; Nichirei, Tokyo, Japan), rabbit anti-Wilms' Tumor Protein polyclonal antibody $(1: 100$; Merck, Darmstadt, Germany) or mouse anti-vimentin monoclonal antibody (1:25, clone V9, Dako, Copenhagen, Denmark) were applied. The immunoreactive signal was visualized by peroxidase-conjugated anti-mouse [Histofine Simple Stain MAX-PO(M); Nichirei] or anti-rabbit [Histofine Simple Stain MAX-PO(R); Nichirei] immunoglobulin (Ig)G and 3,3'-diaminobentidine. Methyl green or Mayer's hematoxylin was used for counterstaining.

WT-1-positive cells were regarded as glomerular podocytes[16]. The number of WT-1-positive cells $\left(\mathrm{N}_{\mathrm{P}}\right)$ was counted for each glomerulus. A total of 50 glomeruli was examined for each kidney section and the podocyte number per glomerular area was described as $\mathrm{N}_{\mathrm{P}} / \mathrm{A}_{\mathrm{G}}$. The vimentin-positive area was measured for each glomerulus using ImageJ and was defined as podocyte area $\left(A_{P}\right)$. A total of 50 glomeruli was examined for podocyte area and damage. Podocyte area per glomerulus was described as $\left(A_{P} / A_{G}\right) \times 100$. Desmin was used as a maker of damaged podocytes [17]. The desmin-positive area $\left(A_{D}\right)$ within a glomerulus was used to provide the desmin score using the formula $\left(\mathrm{A}_{\mathrm{D}} / \mathrm{A}_{\mathrm{G}}\right) \times 100$.

Electron microscopy: Cortical renal tissue from F344 and $\mathrm{OM}$ rats was cut into $1-\mathrm{mm}^{3}$ cubes, fixed in $2.5 \%$ glutaraldehyde, and post-fixed in $1 \% \mathrm{OsO}_{4}$ for $2 \mathrm{hr}$. The fixed specimens were then dehydrated through ascending grades of alcohol and embedded in epoxy resin. Ultra-thin sections were double-stained with uranyl acetate and lead citrate, and a JEOL 1210 transmission electron microscope (JEOL, Tokyo, Japan) at $80 \mathrm{kV}$ was used for examination.

Statistical analysis: Results are expressed as the mean \pm SD. Values within the same strain of rats were compared using an unpaired Student's $t$-test. Comparison between age-matched F344 and OM rats was performed by the Mann-Whitney U-test. Statistical significance was defined as $P<0.05$.

\section{RESULTS}

Blood pressure, serum and urine analysis: As shown in Table 1, systolic blood pressures of OM rats at 7, 13 and 20 weeks of age were $145 \pm 15,158 \pm 4$ and $141 \pm 11 \mathrm{mmHg}$, respectively. Urinary protein excretion showed no agerelated changes in F344 rats (Fig. 1a), but markedly increased with age in OM rats (Fig. 1b, Table 1). In parallel with the changes in urinary total protein in OM rats, the urinary protein:creatinine ratio significantly increased from 7 to 20 weeks of age (Table 1). SDS-PAGE analysis of urine 
Table 1. Results of clinical and laboratory tests of male OM and F344 rats

\begin{tabular}{|c|c|c|c|c|c|c|c|c|c|c|}
\hline \multirow[t]{2}{*}{ Age (weeks) } & \multicolumn{2}{|c|}{5} & \multirow{2}{*}{$\frac{7}{\mathrm{OM}(\mathrm{n}=8)}$} & \multicolumn{2}{|c|}{10} & \multirow{2}{*}{$\frac{13}{\mathrm{OM}(\mathrm{n}=5)}$} & \multicolumn{2}{|c|}{15} & \multicolumn{2}{|c|}{20} \\
\hline & F344 $(n=3)$ & $\mathrm{OM}(\mathrm{n}=6)$ & & F344 $(n=3)$ & $\mathrm{OM}(\mathrm{n}=5)$ & & $\mathrm{F} 344(\mathrm{n}=3)$ & $\mathrm{OM}(\mathrm{n}=7)$ & F344 $(n=3)$ & OM $(n=9)$ \\
\hline Body weight (g) & $77 \pm 13$ & $111 \pm 13^{*}$ & $192 \pm 20$ & $201 \pm 7$ & $295 \pm 17^{*}$ & $361 \pm 7$ & $300 \pm 7$ & $379 \pm 17^{*}$ & $325 \pm 5$ & $382 \pm 48$ \\
\hline SBP (mm Hg) & NA & NA & $145 \pm 15$ & NA & NA & $158 \pm 2.1$ & NA & NA & NA & $141 \pm 11$ \\
\hline Kidney weight (g) & $0.36 \pm 0.03$ & $0.50 \pm 0.06^{*}$ & $0.79 \pm 0.11$ & $0.71 \pm 0.04$ & $1.30 \pm 0.27^{*}$ & $1.75 \pm 0.22$ & $0.94 \pm 0.13$ & $2.26 \pm 0.39 *$ & $0.91 \pm 0.02$ & $2.74 \pm 0.42 *$ \\
\hline \multicolumn{11}{|l|}{ Serum } \\
\hline Total protein $(\mathrm{g} / \mathrm{d} l)$ & $5.2 \pm 0.2$ & $5.5 \pm 0.1$ & $6.1 \pm 0.1$ & $6.1 \pm 0.4$ & $6.1 \pm 0.1$ & $6.4 \pm 0.1$ & $7.1 \pm 0.5$ & $5.8 \pm 0.2$ & $4.4 \pm 2.0$ & $6.2 \pm 0.2$ \\
\hline Albumin $(\mathrm{g} / \mathrm{d} l)$ & $3.7 \pm 0.2$ & $3.8 \pm 0.0$ & $4.0 \pm 0.1$ & $4.1 \pm 0.3$ & $3.6 \pm 0.1$ & $3.5 \pm 0.1$ & $4.8 \pm 0.3$ & $2.9 \pm 0.1$ & $2.9 \pm 1.3$ & $2.8 \pm 0.0$ \\
\hline $\mathrm{BUN}(\mathrm{mg} / \mathrm{d} l)$ & $40 \pm 4$ & $15 \pm 3 *$ & $16 \pm 1$ & $20 \pm 1$ & $20 \pm 1$ & $23 \pm 1$ & $17 \pm 1$ & $29 \pm 4^{*}$ & $12 \pm 3$ & $60 \pm 20^{*}$ \\
\hline Creatinine $(\mathrm{mg} / \mathrm{d} l)$ & $0.5 \pm 0.0$ & $0.6 \pm 0.0$ & $0.7 \pm 0.0$ & $0.5 \pm 0.0$ & $0.7 \pm 0.0$ & $0.8 \pm 0.0$ & $0.5 \pm 0.0$ & $0.8 \pm 0.1 *$ & $0.4 \pm 0.2$ & $1.3 \pm 0.2 *$ \\
\hline Cholesterol $(\mathrm{mg} / \mathrm{d} l)$ & $59 \pm 1$ & $111 \pm 5^{*}$ & $115 \pm 4$ & $43 \pm 5$ & $184 \pm 54 *$ & $240 \pm 26$ & $50 \pm 4$ & $327 \pm 61 *$ & $32 \pm 15$ & $541 \pm 122^{*}$ \\
\hline \multicolumn{11}{|l|}{ Urine } \\
\hline Total protein $(\mathrm{g} / \mathrm{d} l)$ & $0.01 \pm 0.01$ & $0.05 \pm 0.03^{*}$ & $0.30 \pm 0.18$ & $0.08 \pm 0.02$ & $1.12 \pm 0.55^{*}$ & NA & $0.06 \pm 0.02$ & $1.88 \pm 0.57 *$ & $0.08 \pm 0.02$ & $2.59 \pm 1.13^{*}$ \\
\hline Albumin $(\mu \mathrm{g} / \mathrm{m} l)$ & $\mathrm{NA}^{\dagger}$ & $2.8 \pm 0.9$ & $8.1 \pm 5.1$ & $6.0 \pm 0.0$ & $21.6 \pm 8.4^{*}$ & NA & $8.3 \pm 1.1$ & $39.5 \pm 11.9^{*}$ & $15.6 \pm 6.0$ & $55.6 \pm 24.5^{*}$ \\
\hline $\mathrm{UP} / \mathrm{C}$ (mg/mg Cre) & $0.3 \pm 0.0$ & $1.2 \pm 0.9^{*}$ & $3.2 \pm 2.0$ & $1.7 \pm 0.3$ & $10.5 \pm 6.1^{*}$ & $13.0 \pm 1.3$ & $1.0 \pm 0.4$ & $15.0 \pm 5.5^{*}$ & $0.9 \pm 0.1$ & $22.3 \pm 8.5^{*}$ \\
\hline UA/C (mg/g Cre) & NA & $0.06 \pm 0.03$ & $0.08 \pm 0.05$ & $0.12 \pm 0.00$ & $0.21 \pm 0.10^{*}$ & $0.31 \pm 0.04$ & $0.13 \pm 0.02$ & $0.31 \pm 0.12 *$ & $0.17 \pm 0.05$ & $0.48 \pm 0.18^{*}$ \\
\hline
\end{tabular}

Values are mean $\pm \mathrm{SD}$; SBP, systolic blood pressure; NA, not available; UP/C, urinary protein creatinine ratio; UA/C, urinary albumin/creatinine ratio; Cre, Creatinine; * $P<0.05$ versus age-matched F344 rats; $\dagger<5 \mu \mathrm{g} / \mathrm{ml}$.

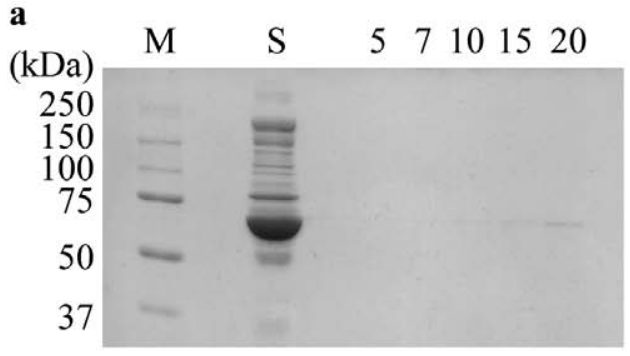

b

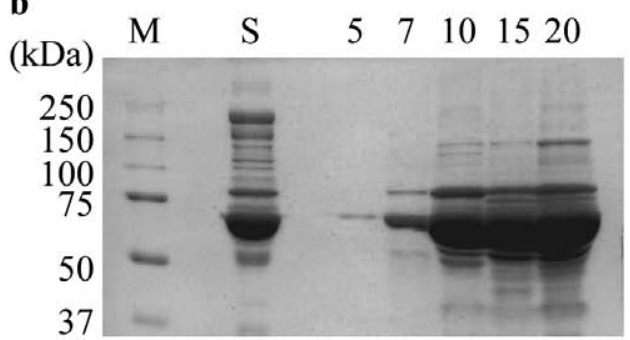

c

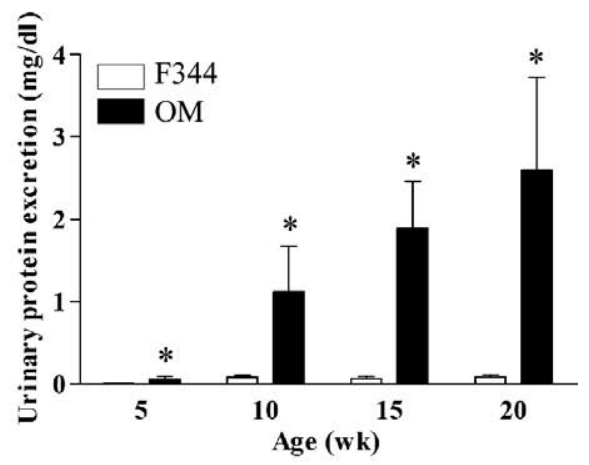

Fig. 1. Urine analysis of F344 and OM rats. SDS-PAGE of urine at 5, 7, 10, 15, and 20 weeks of age of F344 (a) and $\mathrm{OM}$ (b) rats. Lane $\mathrm{M}$ is a standard protein marker and lane $\mathrm{S}$ is normal rat serum. Note the apparent increase in urinary protein excretion from 7 weeks of age in OM rats. The change of urinary protein excretion of F344 and OM rats at 5, 10, 25, 20 weeks of age in $\mathrm{mg} / \mathrm{d} l$ (c). ${ }^{*} P<0.05$ versus F344 rats of the same age.

from $\mathrm{OM}$ rats revealed the presence of selective proteinuria composed mainly of albumin from 5 weeks of age (Fig. 1b). Non-selective proteinuria comprising proteins of larger molecular weight than albumin was observed from 7 weeks of age. This leakage of proteins into urine rapidly increased with age (Table 1). In contrast, notable proteinuria was not observed until 20 weeks of age in F344 rats (Fig. 1a).

BUN and serum creatinine levels were significantly increased in OM rats at 15 and 20 weeks of age (Table 1). Serum total cholesterol (TC) in OM rats was significantly higher than age-matched F344 rats from 5 to 20 weeks of age (Table 1).

Morphological and morphometric analyses of glomeruli: In OM rats at 5 weeks of age, a small number of podocytes were loaded with PAS-positive fine granules (Fig. 2i). At 7 weeks of age, podocytes with vacuolated cytoplasm were apparent (Fig. 2j) and adhesion of the glomerular capillary loop to the Bowman's capsule was occasionally observed (Fig. 2k). Furthermore, focal and segmental sclerosis developed at 15 weeks of age (Fig. $2 \mathrm{~g}$ ) and global glomerulosclerosis occasionally appeared at 20 weeks of age (Fig. $2 \mathrm{~h}$ ). The sclerosis score significantly increased with age in $\mathrm{OM}$ 

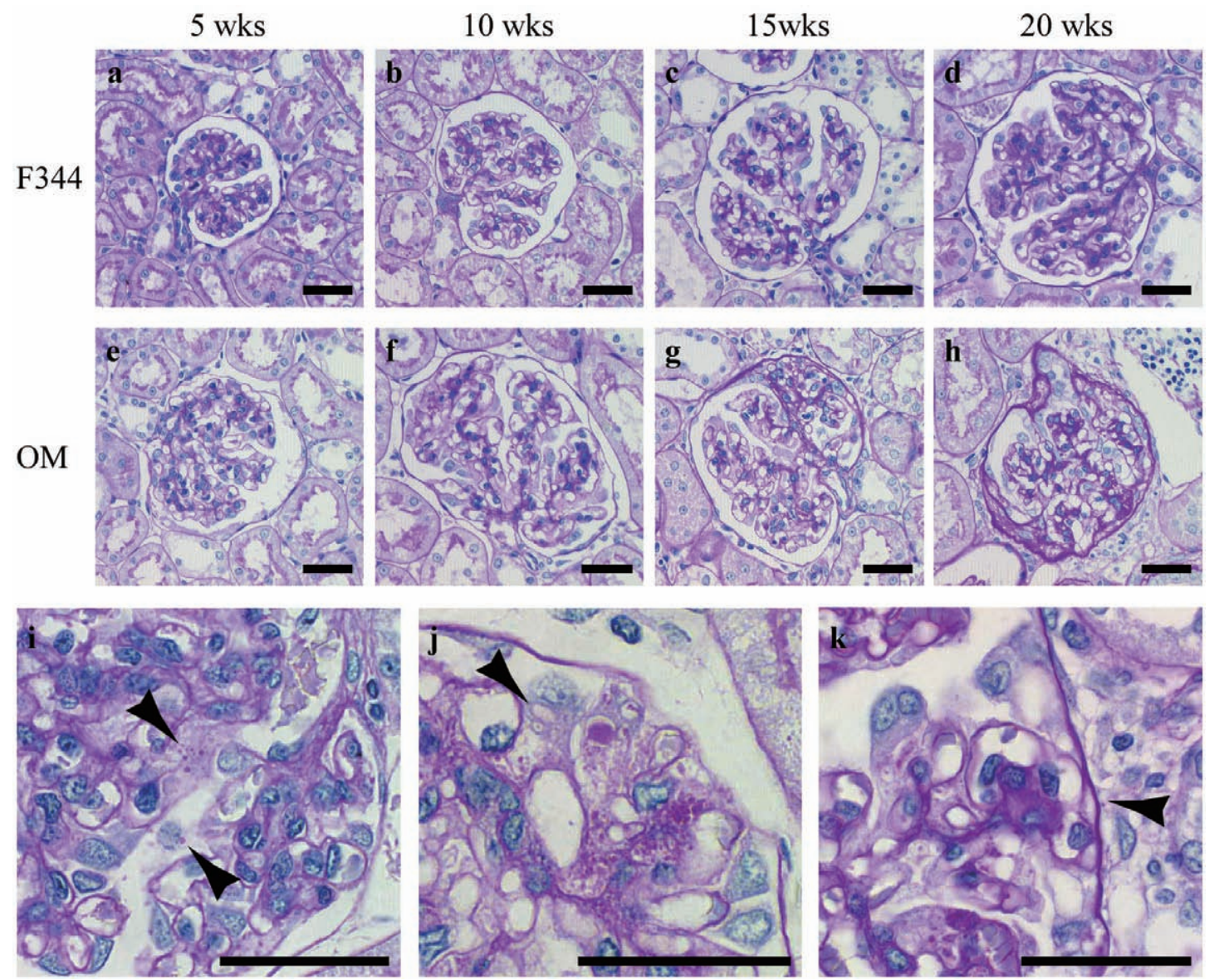

I

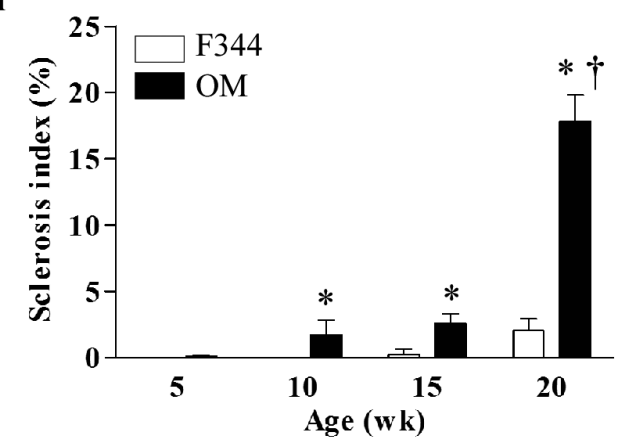

Fig. 2. Glomerular changes and sclerosis scores. Glomerular changes in F344 (a-d) and OM (e-h) rats. Note the difference in glomerular size between the two strains. PAS-positive granules are observed in podocytes in 5 week-old OM rat (arrowheads) (i). Podocyte with small vacuoles in cytoplasm (arrowhead) (j) and glomerular adhesion (arrowhead) (k) in 7 week-old OM rat, scale bar $=30 \mu \mathrm{m}$. Sclerosis index in F344 and OM rats (1). Results are mean $\pm \mathrm{SD}$; * $P<0.05$ versus F344 rats of the same age; ${ }^{\dagger} P<0.05$ versus the 15 week-old rats of the same strain.

rats and in 10,15 and 20 week-old animals was $1.7 \pm 2.2$, $2.6 \pm 1.8$ and $17.8 \pm 1.9 \%$ of glomerulus, respectively (Fig. 21). In contrast, there was no significant renal damage in F344 rats, although a small number of glomeruli showed sclerosis associated with thickening of the GBM and expansion of mesangium at 15 and 20 weeks of age (data not shown).

Interstitial mononuclear cell infiltration appeared at 10 weeks and gradually progressed with age in OM rats (data not shown). Hyaline droplets and/or vacuolar degeneration of the tubular epithelium and formation of hyaline casts were observed from 7 weeks of age. At 15 weeks of age,

tubular atrophy accompanied with thickening of the tubular basement membrane and interstitial fibrosis was present (data not shown).

To evaluate podocyte injury in OM rats, immunostaining for desmin, a conventional marker of podocyte injury[5] , was performed. Desmin was expressed in damaged podocytes and in the mesangial area in both strains of rats (Fig. 3a-d). The desmin score was significantly higher in OM rats than F344 rats from 10 to 20 weeks of age (Fig. 3e). Peak desmin score was observed in OM rats at 10 weeks of age, and then steadily decreased with age.

Glomerular volume and podocyte number: The glomeru- 
F344

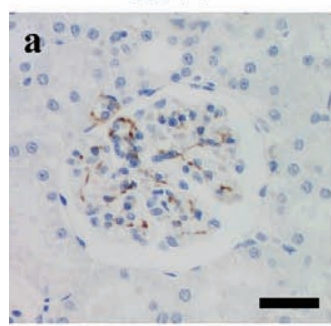

e
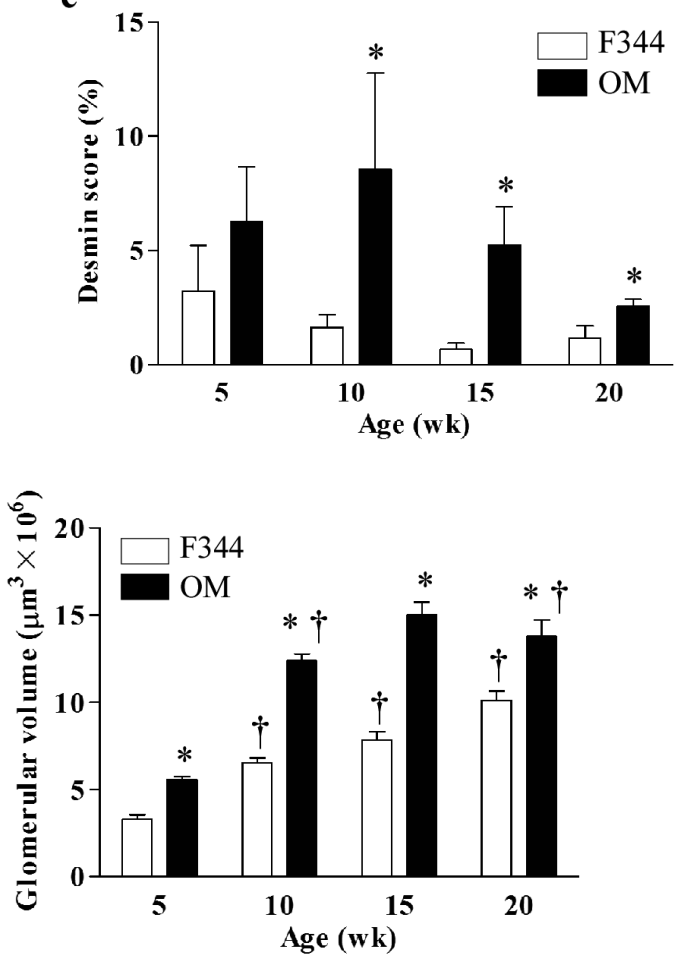

Fig. 4. Glomerular volume in F344 and OM rats. Glomerular volume at 5, 10, 15 and 20 weeks in F344 and OM rats, reported in $\mu \mathrm{m}^{3} \times 10^{6}$. Results are mean $\pm \mathrm{SD}$; $P<0.05$ versus F344 rats of the same age; ${ }^{\dagger} P<0.05$ versus the previous age rats of the same strain.

lar volume $\left(\mathrm{V}_{\mathrm{G}}\right)$ and podocyte density are shown in Figs. 4 and 5. Mean values of $\mathrm{V}_{\mathrm{G}}$ increased with age in both F344 and $\mathrm{OM}$ rats. However, from $5\left(5.6 \pm 0.2 \mu \mathrm{m}^{3} \times 10^{6}\right.$ in $\mathrm{OM}$ rats vs. $3.3 \pm 0.1 \mu \mathrm{m}^{3} \times 10^{6}$ in $\mathrm{F} 344$ rats; $\left.P<0.05\right)$ to 20 weeks of age, $\mathrm{V}_{\mathrm{G}}$ in $\mathrm{OM}$ rats was significantly higher than that in F344 rats (Fig. 4). $\mathrm{V}_{\mathrm{G}}$ in OM rats significantly increased from 5 to 10 weeks of age, and then significantly decreased from 15 to 20 weeks of age (Fig. 4). There was no significant difference in glomerular density between F344 and OM rats $(791.6 \pm 18.4$ in F344 rats vs. $819.2 \pm$ 99.2 in OM rats; $P>0.05)$.

To evaluate the number of podocytes in a glomerulus $\left(\mathrm{N}_{\mathrm{P}}\right)$, the number of WT-1-positive cells was counted and the glomerular area $\left(\mathrm{A}_{\mathrm{G}}\right)$ was measured. As shown in Fig. 5,

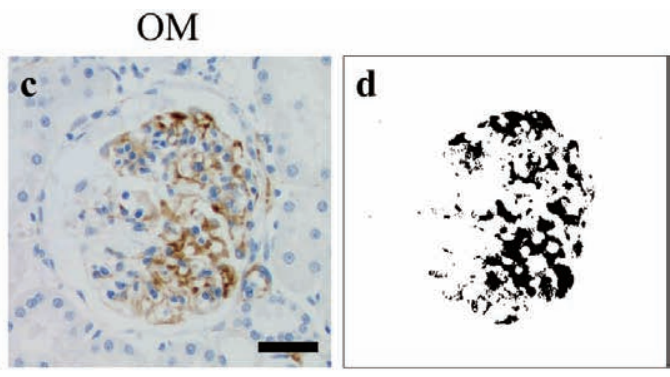

Fig. 3. Expression of desmin and desmin scores in F344 and OM rats. Expression of desmin in F344 $(\mathrm{a}, \mathrm{b})$ and OM (c, d) rats at 10 weeks of age. Desmin immunostain ( $\mathrm{a}, \mathrm{c})$, scale bar $=30 \mu \mathrm{m}$. The digitalized images $(b, d)$ show desmin-positive areas of the same glomerulus. Desmin-positive area per glomerulus $\left(\mathrm{A}_{\mathrm{D}} / \mathrm{A}_{\mathrm{G}}\right)(\mathrm{e})$. Results are mean $\pm \mathrm{SD}$; $P<0.05$ versus $\mathrm{F} 344$ rats of the same age.

WT-1 was specifically localized to the nuclei of podocytes. The number of podocytes per glomerular area $\left(\mathrm{N}_{\mathrm{P}} / \mathrm{A}_{\mathrm{G}}\right)$ gradually decreased from 5 to 20 weeks in OM rats, while podocyte density did not decrease after 10 weeks of age in F344 rats (Fig. $5 \mathrm{~m}$ ). The values of $\mathrm{N}_{\mathrm{P}} / \mathrm{A}_{\mathrm{G}}$ in $\mathrm{OM}$ rats were consistently lower than those of age-matched F344 rats, and this difference was detectable at 5 weeks of age $(3.7 \pm 0.2$ in $\mathrm{OM}$ rats vs. $6.8 \pm 0.3$ in F344 rats; $P<0.05)$.

Podocyte area per glomerular area $\left(\mathrm{A}_{\mathrm{P}} / \mathrm{A}_{\mathrm{G}}\right)$ was estimated using immunostaining for vimentin (Fig. $5 \mathrm{i}-1)$. As vimentin staining is specifically positive for podocytes among the glomerular cells [20], vimentin-positive areas were regarded as the podocyte area in each glomerulus. There was no significant difference between the $A_{P} / A_{G}$ values between rat strains at 5 weeks of age $(9.8 \pm 2.3$ in F344 rats vs. $9.7 \pm 1.8$ in $\mathrm{OM}$ rats; $P<0.05)$. In both rat strains, peak $\mathrm{A}_{\mathrm{P}} / \mathrm{A}_{\mathrm{G}}$ was observed at 10 weeks of age, and steadily decreased with age (Fig. 5n). However, the $A_{P} / A_{G}$ values in $\mathrm{OM}$ rats were significantly lower than in F344 rats after 10 weeks of age $(13.4 \pm 3.6$ in OM rats vs. $20.1 \pm 5.8$ in F344 rats at 10 weeks of age, $5.7 \pm 2.3$ in OM rats vs. $11.5 \pm 1.8$ in F344 rats at 15 weeks of age, $3.3 \pm 1.4$ in OM rats vs. $7.9 \pm$ 0.9 in F344 rats at 20 weeks of age; $P<0.05$ ).

Ultrastructural changes in glomeruli: Effacement and fusion of podocyte foot processes were observed in OM rats as early as 5 weeks of age (Fig. 6b). The numbers of foot processes and filtration slits were greatly reduced with age, and rearrangement of the actin cytoskeleton was observed in fused foot processes in OM rats (Fig. 6b). In addition, the slit pore, which is composed between the foot processes, was narrowed and the slit diaphragm was dislocated in OM rats. The cytoplasm of podocytes contained vacuoles or cytoplasmic pockets in OM rats but not in F344 rats (Fig. $6 \mathrm{c}$ ). Formation of surface microvilli and detachment of 

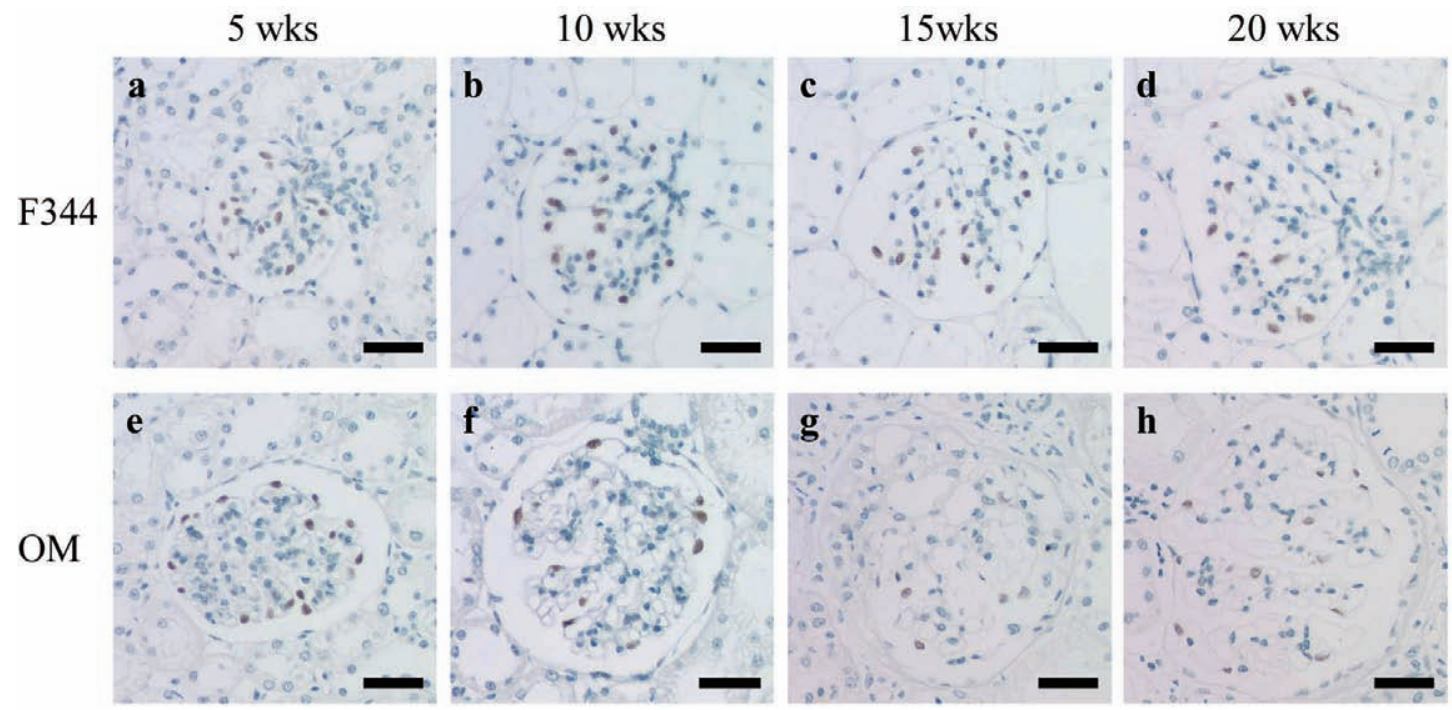

\section{F344}
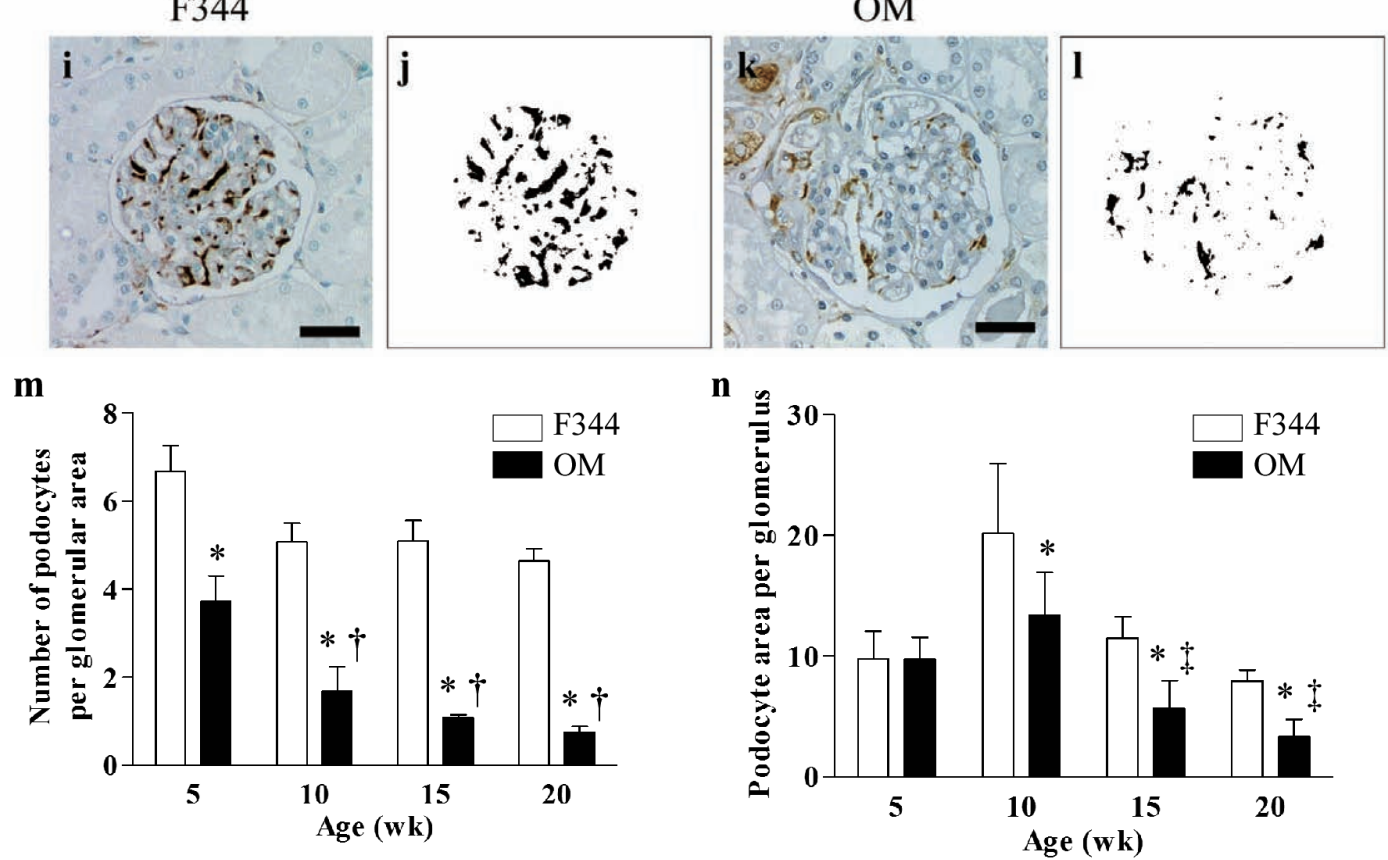

Fig. 5. Podocyte staining, podocyte number, and podocyte area per glomerulus in F344 and OM rats. Expression of WT-1 protein in 5, 10, 15, and 20 week-old F344 (a-d) and OM (e-h) rats, scale bar $=30 \mu \mathrm{m}$. Expression of vimentin in 20 week-old F344 $(\mathrm{i}, \mathrm{j})$ and OM $(\mathrm{k}, \mathrm{l})$ rats. Digitalized images show vimentin-positive areas within the same glomerulus, scale bar $=30 \mu \mathrm{m}$. Podocyte number per glomerular area $\left(\mathrm{N}_{\mathrm{P}} / \mathrm{A}_{\mathrm{G}}\right)(\mathrm{m})$ and podocyte area per glomerulus $\left(\mathrm{A}_{\mathrm{P}} / \mathrm{A}_{\mathrm{G}}\right)(\mathrm{n})$ at $5,10,15$, and 20 weeks of age. Results are mean $\pm \mathrm{SD} ; * P<0.05$ versus F344 rats of the same age; ${ }^{\dagger} P<0.05$ versus the 5 -week-old rats of the same strain; ${ }^{\ddagger} P<0.05$ versus the 10 -week-old rats of the same strain.

degenerated podocytes from the GBM was observed in OM rats at 15 weeks of age. The detachment resulted in denudation of the GBM into the urinary space (Fig. 6d). Mild thickening of the GBM was also detected from 10 to 20 weeks of age in OM rats.

\section{DISCUSSION}

The glomerulopathy of OM rats in the present study was characterized by early development of proteinuria associated with focal and segmental glomerulosclerosis, and followed by tubulointerstitial inflammation and fibrosis. This renal damage had fully developed by 20 weeks of age. Typ- 

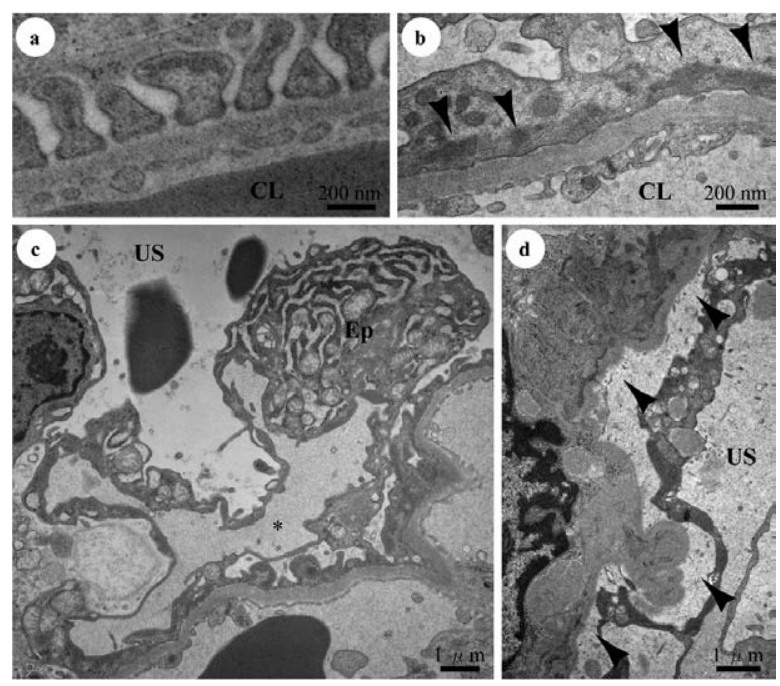

Fig. 6. Transmission electron micrography of podocytes in F344 and $\mathrm{OM}$ rats. Representative electron micrographs of podocytes in a F344 rat at 5 weeks (a), and OM rats at 5 weeks (b) and 15 weeks (c, d). Foot process effacement (b), rearrangement of the actin cytoskeleton (b, arrows), vacuoles in the cytoplasm (c, *), and denudation of the GBM (d, arrows) observed in an OM rat. CL, capillary lumen; Ep, podocyte; US, urinary space.

ical podocytopathies and podocytopenia preceded the development of glomerulosclerosis. These findings indicate that the podocyte damage could be an important factor in the pathogenesis of glomerulonephropathy in OM rats.

Morphologically, mild hyaline droplet degeneration of podocytes and effacement and fusion of foot processes were observed from 5 to 20 weeks, and formation of vacuoles and microvilli were observed from 10 to 20 weeks of age. Ultrastructurally, rearrangement of the actin cytoskeleton, narrowing of slit pores, and displacement of the slit diaphragm were observed in OM rats. These same features have been reported in the previous studies of human and animal podocyte damage[10, 11]. In OM rats, adhesion of the glomerular tufts to the Bowman's capsule and segmental glomerular sclerosis progressed with age from 10 to 20 weeks: the sclerotic areas comprised approximately $20 \%$ of the glomerulus at 20 weeks of age. Non-selective proteinuria in OM rats developed at 7 weeks of age and was associated with apparent podocyte damage. These findings suggest that the development of proteinuria and glomerular lesions in $\mathrm{OM}$ rats is more progressive than the changes reported in other spontaneous animal models of glomerulosclerosis[13, 19]. In addition, podocyte damage as evaluated by desmin expression was significantly more severe in OM rats compared to age-matched F344 rats. The progression of damage to podocytes in OM rats paralleled a decrease in podocyte number within each glomerulus. This podocyte damage preceded glomerular injury, thus suggesting that podocyte damage is important in the pathology of glomerulonephropathy in OM rats.

The spontaneous development of chronic nephropathy in old rats is well documented in many strains, including F344 rats [24]. In previous reports of chronic nephropathy in rats, the morphological changes of glomeruli were characterized by early thickening of the GBM and small mesangial deposits of immunoglobulin [7, 21]. These changes were not observed in the present study of OM rats. Also ultrastructural analyses revealed hypertrophy and fusion of podocyte foot processes, increased formation of protein absorption droplets, and microvillous transformation of cell surface in other rat strains $[7,21]$. These microscopic and ultrastructural changes were similar to those observed in the OM rats in the present study. Most importantly, the onset of nephropathy with proteinuria in the OM rats in this study occurred in much younger rats than that reported with other rat strains.

Glomerular volume progressively increased with age in $\mathrm{OM}$ rats. Although glomerular volumes were consistently lower in age-matched F344 rats than in OM rats, a similar increase in glomerular volume with age was observed. These results suggest that glomerular hypertrophy may develop as early as 5 weeks in OM rats. These glomerular volume changes result in a higher glomerular flow per nephron and yield more filtration. Therefore, they may represent adaptive changes compensating for the inborn nephrons deficits found in younger animals [3, 13]. In the present investigation, the blood pressures of $\mathrm{OM}$ rats were higher than those of reported previously [4]. Systemic hypertension may lead to an increase in intraglomerular pressure, which may be involved in the pathogenesis of glomerular hypertrophy. Further study is needed to clarify the relationship between hypertension and glomerular hypertrophy in OM rats. A decrease in glomerular volumes was detected from 15 to 20 weeks of age in OM rats. This decrease is associated with the increase in sclerotic score at 20 weeks of age. The presence of sclerotic or collapsed glomeruli might reflected the decrease in mean glomerular volume at 20 weeks in $\mathrm{OM}$ rats.

The number of podocytes per glomerulus was evaluated by measuring specific markers of differentiated podocytes in mature glomeruli: WT-1-positive nuclei and the vimentin-positive area [16]. The number of podocytes within each glomerulus was lower in OM rats compared to F344 rats starting at 5 weeks, and the number progressively decreased with age in OM rats. The progressive decrease in podocyte number after 10 weeks of age was not observed in F344 rats. The decrease in podocyte number per glomerular area found between 5 and 10 weeks in both strains could have been due to an increase in glomerular volume associated with physiological nephrogenesis [25]. A remarkable increase in glomerular area might affect the number of podocytes within a glomerulus, and cause the assumed reduction in podocyte number per glomerulus. However, there was a significant difference between the two strains in the number of podocytes per glomerulus from 5 weeks of age. The rate of decrease of podocyte number was also much higher in OM rats.

In some human disease conditions, podocyte nuclei 
become negative for WT-1 as the cells undergo phenotypic dysregulation such as human immunodeficiency virus (HIV) nephropathy and collapsing focal segmental glomerulosclerosis (FSGS) [1]. Therefore, vimentin was also used as a podocyte specific marker to calculate podocyte area. Podocyte area within a glomerulus was highest at 10 weeks of age, and then progressively decreased with age in both F344 and OM rats. The increase in podocyte area between 5 and 10 weeks could be a consequence of the physiological changes associated with podocyte maturation. However, the notable reduction in podocyte area after 10 weeks in OM rats suggests a loss of podocytes within each glomerulus. This reduction in the number of podocytes is also suggested by the decrease in both the WT-1-positive nucleus counts and vimentin-positive area within each glomerulus. These decreases were found before the development of glomerular sclerosis, and suggest that the loss of podocytes may be part of the pathogenesis of glomerular injury in OM rats. Wharram et al. [25] showed that depletion of podocytes by 21 to $40 \%$ resulted in apparent mesangial expansion, synechiae formation, FSGS, and low-level but sustained proteinuria in nephropathy model rats. Moreover, there was a significant correlation between the decrease in podocyte number and development of glomerulosclerosis. In the present study, the number of podocytes in OM rats at 5 weeks of age was $45 \%$ lower than that in F344 rats. This difference may be involved in the subsequent development of glomerular sclerosis and progressive proteinuria. While the pathogenesis of reduced podocyte number in OM rats remains unclear, apoptosis and detachment of podocytes have been reported as an etiology of podocytopenia [15].

In the present study, OM rats had a relative paucity of podocytes compared to F344 rats and an expansion of glomerular volume. Therefore, podocytes in $\mathrm{OM}$ rats might be exposed to higher mechanical stress due to the greater surface area of glomerular capillary loops. It has been shown that a mechanical stess can increase the synthesis of angiotensin II and angiotensin type I receptor in podocytes, and thereby trigger apoptosis through induced TGF- $\beta$ production [2]. Mechanical stress on podocytes caused by glomerular hypertrophy could be one of the pathogenetic factors in the development of podocytopenia in OM rats.

In conclusion, the present study reports significant proteinuria that develops spontaneously with age in male OM rats. This proteinuria is associated with glomerular hypertrophy and progressive reduction in the podocyte number. The renal injury in OM rats begins to develop at 5 weeks of age and is more progressive than that in F344 rats. These findings suggest that the OM rat could be used as a model of spontaneous glomerulosclerosis associated with podocyte injury.

ACKNOWLEDGMENTS. This research was supported by the Promotion and Mutual Aid Corporation for Private Schools of Japan, Grant-in-Aid for Matching Fund Subsidy for Private Universities. We wish to thank Japan SLC, Shizuoka, Japan for providing Osborne-Mendel rats.

\section{REFERENCES}

1. Barisoni, L., Kriz, W., Mundel, P. and D'agati, V. 1999. The dysregulated podocyte phenotype: a novel concept in the pathogenesis of collapsing idiopathic focal segmental glomerulosclerosis and HIV-associated nephropathy. J. Am. Soc. Nephrol. 10: 51-61.

2. Durvasula, R. V., Petermann, A. T., Hiromura, K., Blonski, M., Pippin, J., Mundel, P., Pichler, R., Griffin, S., Couser, W. G. and Shankland, S. J. 2004. Activation of a local tissue angiotensin system in podocytes by mechanical strain. Kidney Int. 65: 30-39.

3. Fassi, A., Sangalli, F., Maffi, R., Colombi, F., Mohamed, E. I., Brenner, B. M., Remuzzi, G. and Remuzzi, A. 1998. Progressive glomerular injury in the MWF rat is predicted by inborn nephron deficit. J. Am. Soc. Nephrol. 9: 1399-1406.

4. Fitzgerald, S. M., Henegar, J. R., Brands, M. W., Henegar, L. K. and Hall, J. E. 2001. Cardiovascular and renal responses to a high-fat diet in Osborne-Mendel rats. Am. J. Physiol. Regul. Integr. Comp. Physiol. 281: R547-552.

5. Floege, J., Hackmann, B., Kliem, V., Kriz, W., Alpers, C. E., Johnson, R. J., Kuhn, K. W., Koch, K. M. and Brunkhorst, R. 1997. Age-related glomerulosclerosis and interstitial fibrosis in Milan normotensive rats: a podocyte disease. Kidney Int. 51: 230-243.

6. Kerjaschki, D. 2001. Caught flat-footed: podocyte damage and the molecular bases of focal glomerulosclerosis. J. Clin. Invest. 108: $1583-1587$.

7. Kihara, I., Yaoita, E. and Yamamoto, T. 1990. Cellular processes of glomerular adhesion in aged rats. Acta Medica. Biol. 38: 69-80.

8. Kim, Y. H., Goyal, M., Kurnit, D., Wharram, B., Wiggins, J., Holzman, L., Kershaw, D. and Wiggins, R. 2001. Podocyte depletion and glomerulosclerosis have a direct relationship in the PAN-treated rat. Kidney Int. 60: 957-968.

9. Kriz, W. 1996. Progressive renal failure-inability of podocytes to replicate and the consequences for development of glomerulosclerosis. Nephrol. Dial. Transplant. 11: 17381742 .

10. Kurihara, H., Anderson, J. M., Kerjaschki, D. and Farquhar, M. G. 1992. The altered glomerular filtration slits seen in puromycin aminonucleoside nephrosis and protamine sulfate-treated rats contain the tight junction protein ZO-1. Am. J. Pathol. 141: 805-816.

11. Lahdenkari, A. T., Lounatmaa, K., Patrakka, J., Holmberg, C., Wartiovaara, J., Kestila, M., Koskimies, O. and Jalanko, H. 2004. Podocytes are firmly attached to glomerular basement membrane in kidneys with heavy proteinuria. J. Am. Soc. Nephrol. 15: 2611-2618.

12. Ly, J., Alexander, M. and Quaggin, S. E. 2004. A podocentric view of nephrology. Curr. Opin. Nephrol. Hypertens. 13: 299 305.

13. Macconi, D., Bonomelli, M., Benigni, A., Plati, T., Sangalli, F., Longaretti, L., Conti, S., Kawachi, H., Hill, P., Remuzzi, G. and Remuzzi, A. 2006. Pathophysiologic implications of reduced podocyte number in a rat model of progressive glomerular injury. Am. J. Pathol. 168: 42-54.

14. Madiehe, A. M., Schaffhauser, A. O., Braymer, D. H., Bray, G. A. and York, D. A. 2000. Differential expression of leptin receptor in high- and low-fat-fed Osborne-Mendel and S5B/P1 rats. Obes. Res. 8: 467-474.

15. Mundel, P. and Shankland, S. J. 2002. Podocyte biology and response to injury. J. Am. Soc. Nephrol. 13: 3005-3015. 
16. Mundlos, S., Pelletier, J., Darveau, A., Bachmann, M., Winterpacht, A. and Zabel, B. 1993. Nuclear localization of the protein encoded by the Wilms' tumor gene WT1 in embryonic and adult tissues. Development 119: 1329-1341.

17. Nagase, M., Shibata, S., Yoshida, S., Nagase, T., Gotoda, T. and Fujita, T. 2006. Podocyte injury underlies the glomerulopathy of Dahl salt-hypertensive rats and is reversed by aldosterone blocker. Hypertension 47: 1084-1093.

18. Nagata, M. and Kriz, W. 1992. Glomerular damage after uninephrectomy in young rats. II. Mechanical stress on podocytes as a pathway to sclerosis. Kidney Int. 42: 148-160.

19. Nakamura, T., Oite, T., Shimizu, F., Matsuyama, M., Kazama, T., Koda, Y. and Arakawa, M. 1986. Sclerotic lesions in the glomeruli of Buffalo/Mna rats. Nephron 43: 50-55

20. Pavenstadt, H., Kriz, W. and Kretzler, M. 2003. Cell biology of the glomerular podocyte. Physiol. Rev. 83: 253-307.

21. Peter, C. P., Burek, J. D. and Van Zwieten, M. J. 1986. Spontaneous nephropathies in rats. Toxicol. Pathol. 14: 91-100.
22. Pittman, D. W., Smith, K. R., Crawley, M. E., Corbin, C. H., Hansen, D. R., Watson, K. J. and Gilbertson, T. A. 2008. Orosensory detection of fatty acids by obesity-prone and obesity-resistant rats: strain and sex differences. Chem. Senses 33: 449-460.

23. Schelling, J. R. and Sedor, J. R. 2004. The metabolic syndrome as a risk factor for chronic kidney disease: more than a fat chance? J. Am. Soc. Nephrol. 15: 2773-2774.

24. Solleveld, H. A. and Boorman, G. A. 1986. Spontaneous renal lesions in five rat strains. Toxicol. Pathol. 14: 168-174.

25. Wharram, B. L., Goyal, M., Wiggins, J. E., Sanden, S. K., Hussain, S., Filipiak, W. E., Saunders, T. L., Dysko, R. C., Kohno, K., Holzman, L. B. and Wiggins, R. C. 2005. Podocyte depletion causes glomerulosclerosis: diphtheria toxin-induced podocyte depletion in rats expressing human diphtheria toxin receptor transgene. J. Am. Soc. Nephrol. 16: 2941-2952.

26. Wiggins, R. C. 2007. The spectrum of podocytopathies: a unifying view of glomerular diseases. Kidney Int. 71: 1205-1214. 